\title{
Comparing VBM and ROI analyses for detection of gray matter abnormalities in patients with bipolar disorder using MRI
}

\author{
Somayyeh Seyedi', Raheleh Jafari ${ }^{2}$, Ali Talaei², Shahrokh Naseri ${ }^{1}$, Mahdi Momennezhad ${ }^{1}$, \\ Maliheh Dadgar Moghaddam ${ }^{3}$ and Hossein Akbari-Lalimi ${ }^{*}$ (D)
}

\begin{abstract}
Background: With the increasing efforts to a better understanding of psychiatric diseases, detection of brain morphological alterations is necessary. This study compared two methods-voxel-based morphometry (VBM) and region of interest $(\mathrm{ROI})$ analyses - to identify significant gray matter changes of patients with bipolar disorder type I (BP I).

Results: The VBM findings suggested gray matter reductions in the left precentral gyrus and right precuneus of the patients compared to healthy subjects ( $a=0.0005$, uncorrected). However, no regions reached the level of significance in $\mathrm{ROI}$ analysis using the three atlases, i.e., hammers, Ipba40, and neuromorphometrics atlases ( $a=$ 0.0005).

Conclusion: It can be concluded that VBM analysis seems to be more sensitive to partial changes in this study. If $\mathrm{ROI}$ analysis is employed in studies to detect structural brain alterations between groups, it is highly recommended to use VBM analysis besides.
\end{abstract}

Keywords: Voxel-based morphometry, ROI analysis, Bipolar disorder, MRI, Brain

\section{Background}

The brain is not a rigid organ, and its structures change by different kinds of experiences and diseases. Localization of structural brain changes on magnetic resonance imaging (MRI) scans is a laborious issue in psychiatric diseases [1, 2]. Many investigators have been using MRI scans as a tool for diagnosis of neurological diseases or tracking disease progression, etc. Therefore, to help them, automated methods have been replaced to identify brain changes without the need for timeconsuming manual measurement, and have grown in popularity since their introduction.

One of these automated methods is voxel-based morphometry (VBM) introduced by Ashburner and

\footnotetext{
* Correspondence: H_Akbari_L@yahoo.com

${ }^{1}$ Medical Physics Department, Faculty of Medicine, Mashhad University of Medical Sciences, Mashhad, Iran

Full list of author information is available at the end of the article
}

Friston [3]. This method is objective and able to perform a voxel-wise estimation to localize changes of a specific tissue. VBM commonly uses T1-weighted MRI scans and performs statistical tests across all voxels in the image to identify volume differences between groups. In VBM, there are three main preprocessing steps before statistical tests: segmentation, normalization, and smoothing.

The first step in preprocessing is segmentation. In this step, gray matter (GM), white matter (WM), cerebrospinal fluid (CSF), and other tissues are extracted. Once an original brain image is used, it is primarily corrected for inhomogeneity of the magnetic field which affects the intensity values of the image voxel. This correction is called bias correction. Another factor that should be well addressed is the partial volume effect. The effect can occur at the boundaries of the tissues whose

\section{Springer Open}

(c) The Author(s). 2020 Open Access This article is licensed under a Creative Commons Attribution 4.0 International License, which permits use, sharing, adaptation, distribution and reproduction in any medium or format, as long as you give appropriate credit to the original author(s) and the source, provide a link to the Creative Commons licence, and indicate if changes were made. The images or other third party material in this article are included in the article's Creative Commons licence, unless indicated otherwise in a credit line to the material. If material is not included in the article's Creative Commons licence and your intended use is not permitted by statutory regulation or exceeds the permitted use, you will need to obtain permission directly from the copyright holder. To view a copy of this licence, visit http://creativecommons.org/licenses/by/4.0/. 
intensity values overlap [4]. By these corrections, the segmented tissue maps are produced.

To compare tissue-segmented images, the images must be normalized. Normalization ascertains that different brain sizes, different head positions, and somewhat different brain shapes of the subjects during $M R$ imaging are corrected using linear and nonlinear normalizations although small differences still remain.

The final step of preprocessing is smoothing. In this step, the normalized segmented images are convolved with an isotropic Gaussian kernel. The output is a weighted average of each voxel's neighborhood. The underlying reasons for using smoothing are an increase of normality of residuals and signal to noise ratio and decrease of effect of misregistration between images [5].

After preprocessing, statistical analysis is performed on the images. It can be parametric using general linear model [3] or nonparametric [6, 7]. A statistical test demonstrates alterations in tissue volume between subject groups to a user-selected $p$ value. To remove false positives from the results, some methods such as family-wise error (FWE) correction or false discovery rate (FDR) correction could be applied $[8,9]$. The final result is a statistical map localizing differences of a specified tissue between groups.

Three approaches of VBM include standard, optimized, and DARTEL (Diffeomorphic Anatomic Registration Through Exponentiated Lie algebra algorithm) [10-12]. The three approaches of VBM have been described in the literatures in detail $[13,14]$. The difference between DARTEL and two first approaches is that using DARTEL, the high dimensional wrapping process was performed [13]. Therefore, misregistration and inaccuracies are reduced more between the template and individual images as well as credibility of the research is increased $[15,16]$.

The other method is an automated ROI analysis [17]. To perform this analysis, probabilistic brain atlases are employed. Probabilistic atlasing is a technique that generates anatomical templates and retains quantitative information on inter-subject variations across the population used to construct the atlas [18]. Using these atlases, it may solve problems of manual ROI assessment and increase repeatability of studies. Examples of these atlases are hammers, lpba40, and neuromorphometrics which are described below.

The three atlases are created using a label-based approach and based on multiple subjects. They are created using manual tracing on anatomical MRI from healthy subjects. The individual subject classifications are then registered to MNI space to generate a probabilistic atlas. The hammers, lpba40, and neuromorphometrics are composed of 69, 40, and 140 regions, respectively. These regions cover the whole cortex and the main subcortical structures. The probabilistic brain atlases have been detailed in the literatures [19-21].

More recently, the abovementioned automatic methods are being increasingly applied to detect the brain volumetric alterations [22] in psychiatric diseases such as Alzheimer's disease [23, 24], epilepsy [25], Parkinson's disease [26], and bipolar disorder [27, 28].

In this regard, Lagopoulos et al. found that there were potential changes in the WM content of the corpus callosum of BP I patients in the early stage of the disease using structural MRI and DTI and FSL software [29]. Several investigations indicated the WM and GM changes in different parts of BP patients' brains including the amygdala, hippocampus, and temporal and frontal lobes [30, 31]. Also, Mahon et al. proposed that deficits in dorsolateral prefrontal and limbic cortical structures were the main manifestations of BP disorder [32].

The present study had three objectives. The primary aim was to apply DARTEL VBM to detect structural GM changes in patients with BP I in comparison to the healthy group. The second aim was to compare the three probabilistic brain atlases, i.e., hammers, lpba40, and neuromorphometrics atlases. The final aim of this study was to assess these methods, i.e., VBM versus ROI analyses. It is hypothesized that a VBM analysis of the same data would complement the ROI findings. In the present study, we used Computational Anatomy Toolbox (CAT12) which is an extension to the SPM12 software package (Statistical Parametric Mapping).

\section{Methods}

\section{Subjects}

The subjects of the present study were 25 patients and 25 healthy people. It was conducted February 2017 to December 2018. Patients with BP I were selected by interview based on DSM-IV-TR criteria, direct assessment by two psychiatrists and medical records. Subjects were excluded if they had a history of substance misuse, neurological disease, or closed head injury. All patients were at their late remissions. They took lithium/valproate and antipsychotic medication. The most and the least numbers of the episodes were 17 and 1, respectively. The median number of episodes was 2 .

The healthy group was included from a pool of community volunteers and assessed with the same criteria as the patient group as well as a lack of family psychiatric history. Table 1 summarizes details of the demographic characteristics of the patient and healthy groups. Written informed consent was obtained from all participants, and the study was approved by the local ethics committee. 
Table 1 Demographic characteristics of participants

\begin{tabular}{lll}
\hline Group & Age (years) (mean \pm SD) & Female/male \\
\hline $\mathrm{HG}^{\mathrm{a}}$ & $34.48 \pm 8.32$ & $19 / 6$ \\
$\mathrm{PG}^{\mathrm{b}}$ & $37.68 \pm 10.88$ & $18 / 7$ \\
\hline
\end{tabular}

${ }^{\mathrm{a}}$ Healthy group

${ }^{\mathrm{b}}$ Patient group

\section{MRI acquisition}

High-resolution T1-weighted structural MR images were acquired at Qaem Hospital, Mashhad, Iran, using a 1.5$\mathrm{T}$ symphony scanner (Siemens, Erlangen, Germany) with MP RAGE sequence $(\mathrm{TR}=2300 \mathrm{~ms}$, $\mathrm{TE}=2.98 \mathrm{~ms}$, flip angle $=98^{\circ}$, field of view $=256 \mathrm{~mm} \times 256 \mathrm{~mm} \times 170 \mathrm{~mm}$, acquisition matrix $=256 \times 256$, slice thickness $=1.27$ $\mathrm{mm}$ ) and the Digital Imaging and Communications in Medicine (DICOM) format.

\section{Voxel-based morphometry}

For VBM analysis, the CAT12 toolbox implemented in SPM12 software was employed. The software was run in MATLAB version 9.3 (The MathWorks, MA, USA). All 3D T1-weighted MR images were converted into the Neuroimaging Informatics Technology Initiative (NIFTI) format through SPM12. The images were spatially normalized and segmented into GM, WM, and CSF tissue classes according to the DARTEL approach with default settings in $1.5 \mathrm{~mm}$ cubic resolution and MNI space. The normalized maps were modulated with the resulting Jacobian determinant maps to preserve GM volumes of native space and smoothed with an 8-mm FWHM Gaussian kernel. The steps of segmentation, normalization, and modulation were automatically done in tandem in the CAT12 toolbox. Total intracranial volume (TIV) and the native space volumes of GM, WM, and CSF maps were estimated as well.

In order to compare the results with ROI results, the GLM analysis was used with TIV as a covariate of no interest because, in ROI analysis, the effect of TIV was corrected. The two-tailed $t$ test was then generated using family-wise error (FWE) correction with a $p<0.05$ and additionally with uncorrected $p<$ 0.0005 thresholds. The extent threshold was set at 100 voxels. The processing framework of VBM analysis is shown in Fig. 1.

\section{ROI analysis}

Using CAT12, regional tissue volumes were estimated in different regions based on the probabilistic atlases. All volumes are approximated in their native space using a high-dimensional spatial registration before any spatial normalization. By extracting data, GM volumes of different structures were determined. To remove the effect of variations in brain sizes, GM volumes of different structures were divided into TIV of the related subject, and then, the GM ratio of each region was obtained. The Mann-Whitney $U$ test was used at the significance level of $0.05 \%$ (i.e., $\alpha=0.0005$, Bonferroni correction) for comparison of two groups using SPSS software, version 16 (IBM-SPSS, Armonk, NY, USA).

\section{Results \\ The VBM analysis}

In voxel by voxel analysis, no region showed significant alteration in healthy controls versus patients using FWE with $p$ value $<0.05$ in the $t$ test. Nonetheless, when an uncorrected $p$ value $<0.0005$ was applied, two regions demonstrated lower GM ratios in the patients compared to the healthy subjects in the two-tailed $t$ test. It should be indicated that when the contrast, patients > healthy subjects, was selected, no brain regions exhibited significant alterations in the patients over the healthy controls. Figure 2 and Table 2 detail the related regions and MNI coordinates of the peak voxels.

\section{ROI analyses}

To compare the results of the ROI with those of VBM, the significant level of $\alpha<0.0005$ was selected. None of the probabilistic brain atlases demonstrated a significant difference in GM ratios between the two groups.

\section{Discussion \\ Within VBM analysis}

We performed a two-tailed $t$ test with a covariate of no interest (i.e., TIV) and compared the bipolar patients over the healthy controls in Table 2 . Using $p<0.05$ corrected, VBM analysis indicated no significant changes in GM volumes of the patients compared to those of the healthy subjects. The reverse contrast had the same result, as well. While the bipolar patients showed a significantly lower volume of GM in the left precentral gyrus and right precuneus than the healthy subjects, no region was higher in the patients than the controls using $p<$ 0.0005 uncorrected and extent threshold of 100 .

To compare our VBM results with other studies' results, it should be noted that the results of VBM analyses of bipolar disorder are contradictory. Some studies reported no significant differences in gray matter volumes between patients and healthy subjects [33, 34] while other studies indicated alterations in different regions of the brain such as frontal gyrus [35, 36], and temporal and parietal gyrus [37]. Besides, no study could replicate the same findings of previous studies. The reason for this may stem from using different procedures, thresholds, kernels, sample size, and statistical corrections, as well as different inclusion criteria. Another reason can be that perhaps there are different subgroups in BP I, which have the same clinical manifestation but different mechanisms and origins. Overall, reported abnormalities 


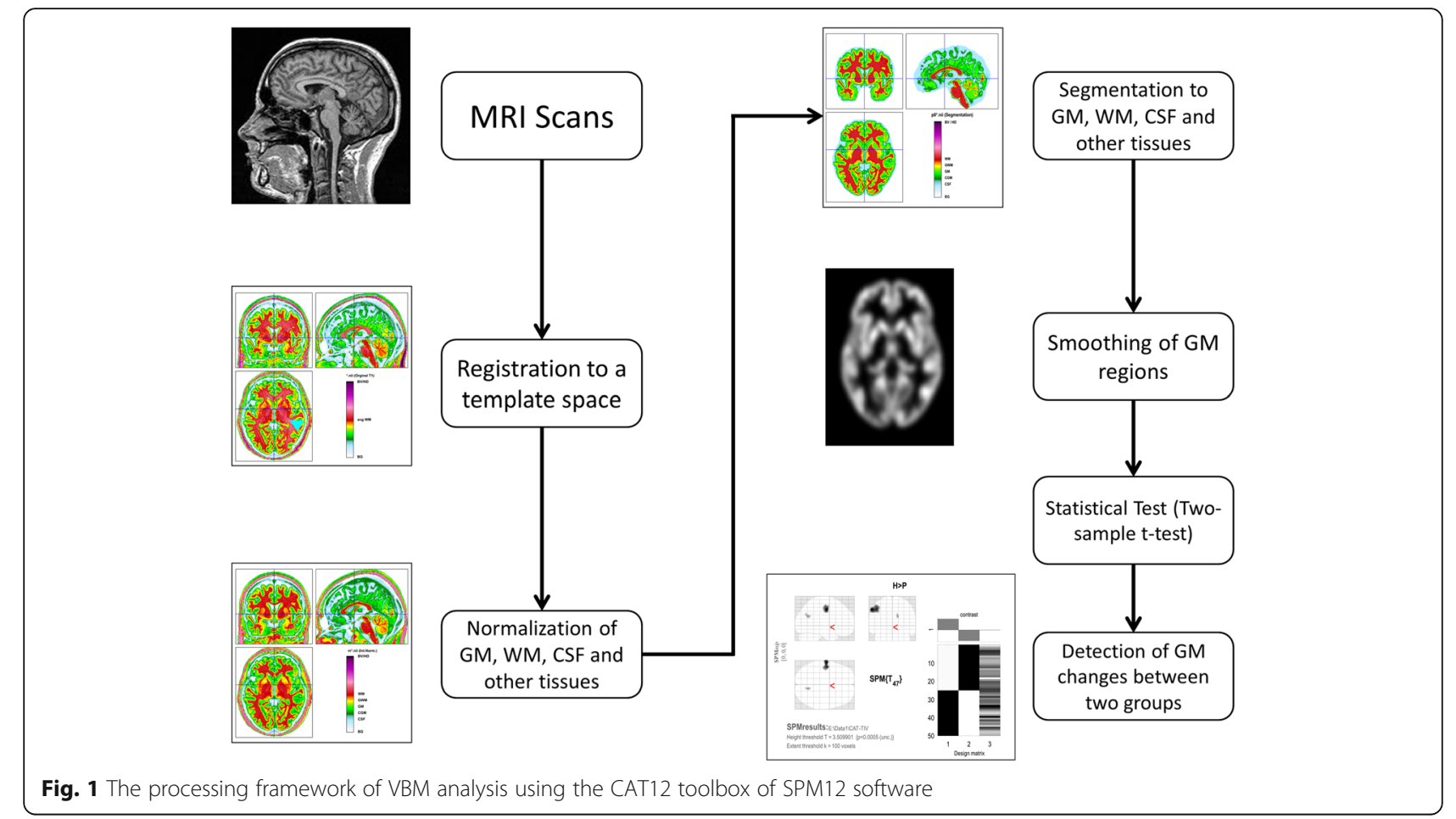

of gray matter volumes are highly dispersed in bipolar disorder.

Taken all together, our VBM results are somewhat similar to the fMRI study in which abnormalities in the precuneus has been reported [38]. In the mentioned study, it was implicated that patients with bipolar disorder showed less activation posterior cingulate cortex/ precuneus compared to healthy controls. The precuneus is responsible for a wide range of cognitive functions including recollection and memory, integration of information related to the perception of the environment, cue reactivity, mental imagery strategies, episodic memory retrieval, and affective responses to pain [39]. The alteration in the functions of the precuneus may alter self-perception as well as the perception of the environment, resulting in behavioral changes that are evident in different episodes of BP.

Also, Eker et al. mentioned a gray matter deficit in patients with bipolar disorder in comparison to unrelated healthy subjects in the left precentral gyrus but right precuneus [40]. The precentral gyrus is the anatomical location of the primary motor cortex, responsible for the control of voluntary movement [41]. GM changes in the precentral gyrus may affect the primary motor cortex function and therefore cause less control on voluntary movement, as can be seen in BP patients.
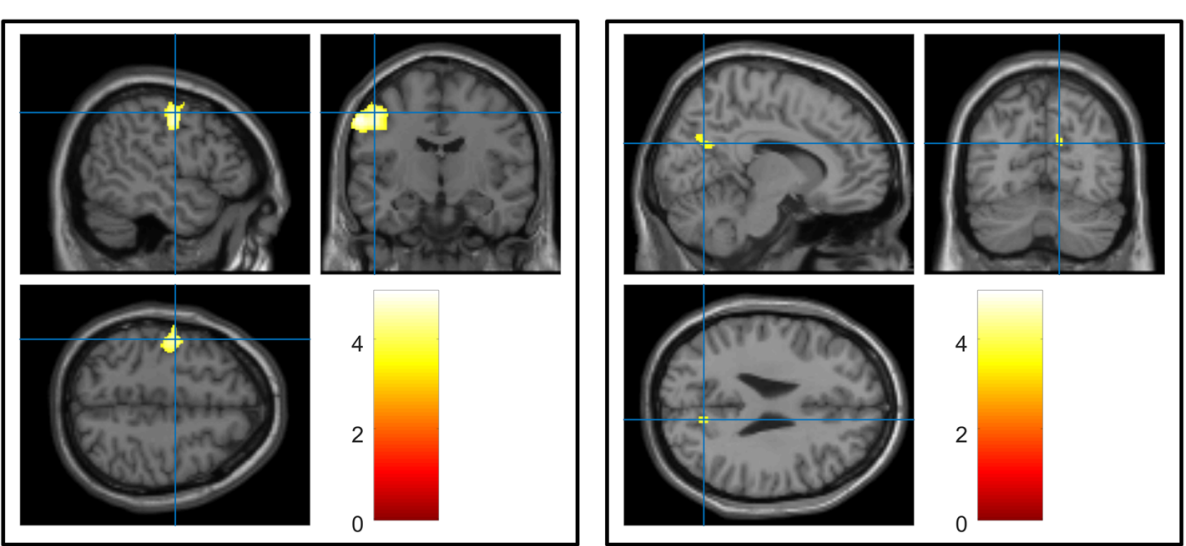

Fig. 2 The significant GM alterations revealed by VBM analyses with the covariate of no interest (TIV) in the a left precentral gyrus and $\mathbf{b}$ right precuneus when HG > PG. $p$ value $<0.0005$ (uncorrected) and extent threshold $K=100$ 
Table 2 GM alterations detected by VBM

\begin{tabular}{|c|c|c|c|c|c|c|c|}
\hline \multirow[t]{2}{*}{$P$ value } & \multirow[t]{2}{*}{ Contrast } & \multirow{2}{*}{$\begin{array}{l}\text { Location of the } \\
\text { peak values }\end{array}$} & \multirow{2}{*}{$\begin{array}{l}\text { Cluster } \\
\text { size } \\
\text { (no. of } \\
\text { voxels) }\end{array}$} & \multicolumn{3}{|c|}{ MNI coordinates } & \multirow{2}{*}{$\begin{array}{l}t \\
\text { value } \\
\text { of the } \\
\text { peak } \\
\text { voxels }\end{array}$} \\
\hline & & & & $X(\mathrm{~mm})$ & $Y(\mathrm{~mm})$ & $Z(\mathrm{~mm})$ & \\
\hline \multirow[t]{2}{*}{$P<0.05$ corr. } & $H G^{a}>P G^{b}$ & - & - & - & - & - & - \\
\hline & $H G<P G$ & - & - & - & - & - & - \\
\hline \multirow[t]{3}{*}{$P<0.0005$ uncorr. } & $H G>P G$ & Left precentral gyrus & 1204 & -61.5 & -10.5 & 43.5 & 5.115 \\
\hline & & Right precuneus & 122 & 13.5 & -63 & 22.5 & 3.949 \\
\hline & $H G<P G$ & - & - & - & - & - & - \\
\hline
\end{tabular}

a Healthy group

${ }^{\mathrm{b}}$ Patient group

\section{Within ROI analysis}

ROI-based analyses were conducted using the three probabilistic brain atlases. There were no regions reaching a significant level. However, the detected regions by VBM come to appear when $p$ value increased to 0.02 . Here, it should be noted that these atlases had several brain region labels such that some labels were similar but the other labels were different.

\section{VBM versus ROI}

One of the aims of this research was to compare the results of VBM to the results of the ROI analysis on the same dataset. For comparison, in VBM, a two-tailed $t$ test with TIV covariate of no interest was employed because replicating the main effects on ROI analysis was interested.

While VBM analysis found two regions of lower GM ratios-namely the left precentral gyrus and right precuneus-in the patient group in comparison to the healthy group, the ROI analysis showed no difference in GM ratios between the two groups.

The reasons for these different results may stem from methodological differences between VBM and ROI methods, which can affect the results. Here, we have discussed it briefly.

In ROI analysis using a probabilistic atlas, an individual brain image was transformed and compared to the atlas as a template. This transformation may cause differences between the image and multiple images constructing the atlas. On the other hand, in a diseased population, local individual brain regions are highly variable, and thus, smaller regions or unusual conformation patterns are more subject to error when transforming. Therefore, appearing and vanishing of some differences may be caused due to inappropriate registration to the atlas.

In contrast to ROI analyses, VBM analysis conducted with CAT12 using the DARTEL approach enjoys precise registrations of images to the template to decrease misregistrations and inaccuracies. Although employing
DARTEL does not yield a perfect registration, many differences due to misregistrations vanish and original anatomical alterations are coded. Furthermore, the selection of the level of significance and extend threshold are two factors that can have an effect on the results.

Another explanation for this difference is that in VBM, we search for differences in the image voxel by voxel rather than one region as a whole just like in ROI analysis. Consequently, if part of a region had a mild to moderate GM differences, this region might not reach a significant level in ROI analysis because the region might have the normal GM volume as a whole. The volume of precentral gyrus, for instance, is 6011 voxels in neuromorphometrics atlas, but the volume of the alteration detected by VBM in this region is 1204 voxels. It means that the volume of the alteration is less than $25 \%$ of the overall volume. Therefore, such a small change may not be detected by the atlas. But as it could be seen in VBM, the analysis is able to detect partial abnormalities even in one region due to voxel by voxel search.

The two methods-VBM and ROI analyses-have advantages and limitations. VBM seems to succeed in the detection of partial differences in GM ratios. However, ROI analysis using the mentioned atlases may be more successful to detect moderate to severe GM abnormalities.

To our knowledge, this study is one of the first studies in patients with BP I using the CAT12 toolbox. But this study had some limitations. One of the limitation was the number of patients with BP I available during research. Another one was the lack of accessibility to the dataset with predefined GM abnormalities. Hence, it is suggested that the similarity between the results of the two methods is investigated by the structural MR images with predefined GM changes with different degrees in severity.

\section{Conclusion}

We performed VBM and ROI analyses to detect brain changes in bipolar patients. DARTEL procedure and 
three probabilistic atlases are used. VBM could detect small changes. Therefore, it can be concluded that VBM analysis seems to be more sensitive to partial changes in this study. If ROI analysis is employed in studies to detect structural brain alterations between groups, it is highly recommended to use VBM analysis besides.

As mentioned, the results of the studies were dispersed for bipolar disorder. The result of this study emphasized it too. The divergence between the results highlighted the necessity of the design of more comprehensive research about bipolar disorder to take into account more psychiatric factors.

\section{Abbreviations \\ VBM: Voxel-based morphometry; ROI: Region of interest; BP I: Bipolar disorder type I; CAT12: Computational Anatomy Toolbox; SPM12: Statistical Parametric Mapping; MRI: Magnetic resonance imaging; GM: Gray matter; WM: White matter; CSF: Cerebrospinal fluid; DARTEL: Diffeomorphic Anatomic Registration Through Exponentiated Lie algebra algorithm; TIV: Total intracranial volume; FWE: Family-wise error; HG: Healthy group; PG: Patient group}

\section{Acknowledgements}

Not applicable.

\section{Authors' contributions}

AT and RJ contributed to assessing participants according to the mentioned criteria, taking MR imaging of them, and giving consultation in terms of psychiatric issues. MD contributed as a statistical advisor. MM and $\mathrm{SH}$. N contributed as advisors in terms of technical issues (image processing and analyzing). HA contributed as a research assistant as well as a technical advisor. SS was a major contributor to image analyzing and writing the manuscript. The author(s) read and approved the final manuscript.

\section{Funding}

The Research Deputy of Mashhad University of Medical Sciences financially supported this research in terms of M.Sc. dissertation.

\section{Availability of data and materials}

The datasets used and/or analyzed during the current study are available from the corresponding author on reasonable request.

\section{Ethics approval and consent to participate}

Written informed consent was obtained from all participants, and the study was approved by the ethics committee of Mashhad University of Medical Sciences with Ref No. IR.MUMS.sm.1396.509.

\section{Consent for publication}

Not applicable.

\section{Competing interests}

The authors declare that they have no competing interests.

\section{Author details}

${ }^{1}$ Medical Physics Department, Faculty of Medicine, Mashhad University of Medical Sciences, Mashhad, Iran. ${ }^{2}$ Psychiatry and Behavioral Sciences Research Center, Mashhad University of Medical Sciences, Mashhad, Iran. ${ }^{3}$ Department of Community Medicine, Faculty of Medicine, Mashhad University of Medical Sciences, Mashhad, Iran.
Received: 29 September 2020 Accepted: 24 November 2020

Published online: 09 December 2020

\section{References}

1. Kubicki M, Shenton ME, Salisbury D et al (2002) Voxel-based morphometric analysis of gray matter in first episode schizophrenia. Neuroimage 17:17111719

2. McDonald C, Zanelli J, Rabe-Hesketh S et al (2004) Meta-analysis of magnetic resonance imaging brain morphometry studies in bipolar disorder. Biol Psychiatry 56:411-417

3. Ashburner J, Friston KJ (2000) Voxel-based morphometry-the methods. Neuroimage 11:805-821

4. Tohka J, Zijdenbos A, Evans A (2004) Fast and robust parameter estimation for statistical partial volume models in brain MRI. Neuroimage 23:84-97

5. Farokhian F, Beheshti I, Sone D et al (2017) Comparing CAT12 and VBM8 for detecting brain morphological abnormalities in temporal lobe epilepsy. Front Neurol 8:428

6. Nichols TE, Holmes AP (2002) Nonparametric permutation tests for functional neuroimaging: a primer with examples. Hum Brain Mapp 15:1-25

7. Rorden C, Bonilha L, Nichols TE (2007) Rank-order versus mean based statistics for neuroimaging. Neuroimage 35:1531-1537

8. Friston KJ, Worsley KJ, Frackowiak RS et al (1994) Assessing the significance of focal activations using their spatial extent. Hum Brain Mapp 1:210-220

9. Genovese CR, Lazar NA, Nichols T (2002) Thresholding of statistical maps in functional neuroimaging using the false discovery rate. Neuroimage 15:870878

10. Colloby SJ, Elder GJ, Rabee R et al (2017) Structural grey matter changes in the substantia innominata in Alzheimer's disease and dementia with Lewy bodies: a DARTEL-VBM study. Int J Geriatr Psychiatry 32:615-623

11. Lochhead RA, Parsey RV, Oquendo MA et al (2004) Regional brain gray matter volume differences in patients with bipolar disorder as assessed by optimized voxel-based morphometry. Biol Psychiatry 55:1154-1162

12. Rajagopalan V, Yue GH, Pioro EP (2014) Do preprocessing algorithms and statistical models influence voxel-based morphometry (VBM) results in amyotrophic lateral sclerosis patients? A systematic comparison of popular VBM analytical methods. J Magn Reson Imaging 40:662-667

13. Ashburner J (2007) A fast diffeomorphic image registration algorithm. Neuroimage 38:95-113

14. Senjem ML, Gunter JL, Shiung MM et al (2005) Comparison of different methodological implementations of voxel-based morphometry in neurodegenerative disease. Neuroimage 26:600-608

15. Klein A, Andersson J, Ardekani BA et al (2009) Evaluation of 14 nonlinear deformation algorithms applied to human brain MRI registration. Neuroimage 46(3):786-802

16. Komatsu J, Matsunari I, Samuraki M et al (2018) Optimization of DARTEL settings for the detection of Alzheimer disease. Am J Neuroradiol 39(3):473478

17. Lao Z, Shen D, Xue Z et al (2004) Morphological classification of brains via high-dimensional shape transformations and machine learning methods. Neuroimage 21:46-57

18. Mazziotta JC, Toga AW, Evans A et al (1995) A probabilistic atlas of the human brain: theory and rationale for its development. Neuroimage 2:89_ 101

19. Gousias IS, Rueckert D, Heckemann RA et al (2008) Automatic segmentation of brain MRls of 2-year-olds into 83 regions of interest. Neuroimage 40:672684

20. Hammers A, Allom R, Koepp MJ et al (2003) Three-dimensional maximum probability atlas of the human brain, with particular reference to the temporal lobe. Hum Brain Mapp 19:224-247

21. Shattuck DW, Mirza M, Adisetiyo V et al (2008) Construction of a 3D probabilistic atlas of human cortical structures. Neuroimage 39:1064-1080

22. Nowinski WL (2016) Usefulness of brain atlases in neuroradiology: current status and future potential. Neuroradiol J 29:260-268

23. Wang W-Y, Yu J-T, Liu Y et al (2015) Voxel-based meta-analysis of grey matter changes in Alzheimer's disease. Transl Neurodegener 4:6

24. Giuliani NR, Calhoun VD, Pearlson GD et al (2005) Voxel-based morphometry versus region of interest: a comparison of two methods for analyzing gray matter differences in schizophrenia. Schizophr Res 74:135147

25. Salgado P, Yasuda C, Cendes F (2012) A voxel based morphometry (VBM) analysis of gray matter volume (GMV) loss in patients with refractory mesial 
temporal lobe epilepsy (MTLE) with and without depression. Epilepsy Behav 24:185

26. Akio H, Togao O, Yamashita K et al (2017) Structural changes in Parkinson's disease: voxel-based morphometry and diffusion tensor imaging analyses based on 123I-MIBG uptake. Eur Radiol 27:5073-5079

27. Ambrosi E, Rossi-Espagnet MC, Kotzalidis GD et al (2013) Structural brain alterations in bipolar disorder II: a combined voxel-based morphometry (VBM) and diffusion tensor imaging (DTI) study. J Affective Disord 150:610615

28. Maggioni E, Crespo-Facorro B, Nenadic I et al (2017) Common and distinct structural features of schizophrenia and bipolar disorder: the European Network on Psychosis, Affective disorders and Cognitive Trajectory (ENPA CT) study. PLoS One 12:e0188000

29. Lagopoulos J, Hermens DF, Hatton SN et al (2013) Microstructural white matter changes in the corpus callosum of young people with bipolar disorder: a diffusion tensor imaging study. PLoS One 8(3):e59108

30. Anderson D, Ardekani BA, Burdick KE et al (2013) Overlapping and distinct gray and white matter abnormalities in schizophrenia and bipolar I disorder. Bipolar Disord 15(6):680-693

31. Craddock N, O'Donovan MC, Owen MJ (2006) Genes for schizophrenia and bipolar disorder? Implications for psychiatric nosology. Schizophr Bull 32(1): 9-16

32. Mahon K, Burdick KE, Szeszko PR (2010) A role for white matter abnormalities in the pathophysiology of bipolar disorder. Neurosci Biobehav Rev 34(4):533-554

33. Bruno S, Barker G, Cercignani M et al (2004) A study of bipolar disorder using magnetization transfer imaging and voxel-based morphometry. Brain 127:2433-2440

34. McIntosh AM, Job DE, Moorhead TWJ et al (2004) Voxel-based morphometry of patients with schizophrenia or bipolar disorder and their unaffected relatives. Biol Psychiatry 56:544-552

35. Lyoo IK, Kim MJ, Stoll AL et al (2004) Frontal lobe gray matter density decreases in bipolar I disorder. Biol Psychiatry 55:648-651

36. Matsuo K, Kopecek M, Nicoletti M et al (2012) New structural brain imaging endophenotype in bipolar disorder. Mol Psychiatry 17:412

37. Doris A, Belton E, Ebmeier KP et al (2004) Reduction of cingulate gray matter density in poor outcome bipolar illness. Psychiatry Res Neuroimaging 130:153-159

38. Zhang L, Opmeer EM, Ruhé HG et al (2015) Brain activation during self-and other-reflection in bipolar disorder with a history of psychosis: comparison to schizophrenia. Neurolmage Clin 8:202-209

39. Zigmond MJ, Coyle JT, Rowland LP (2015) Neurobiology of brain disorders: biological basis of neurological and psychiatric disorders. London: Academic

40. Eker C, Simsek F, YIlmazer EE et al (2014) Brain regions associated with risk and resistance for bipolar I disorder: a voxel-based MRI study of patients with bipolar disorder and their healthy siblings. Bipolar Disord 16:249-261

41. Stern LZ, Bernick C (1990) The Motor System and Gait. In: Walker HK, Hall WD, Hurst JW, editors. Clinical Methods: The History, Physical, and Laboratory Examinations. 3rd ed. Boston: Butterworths. Chapter 68. PMID: 21250232

\section{Publisher's Note}

Springer Nature remains neutral with regard to jurisdictional claims in published maps and institutional affiliations.

\section{Submit your manuscript to a SpringerOpen ${ }^{\circ}$ journal and benefit from:}

- Convenient online submission

- Rigorous peer review

- Open access: articles freely available online

- High visibility within the field

- Retaining the copyright to your article

Submit your next manuscript at $\boldsymbol{\nabla}$ springeropen.com 